\title{
Mean-Field Model Analysis of Deformation and Damage in Friction Stir Processed Mg-C Composites
}

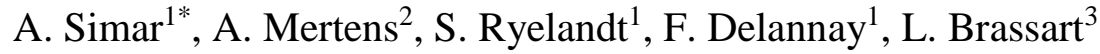 \\ ${ }^{1}$ Institute of Mechanics, Materials and Civil Engineering, Université catholique de Louvain, 2 \\ Place Sainte Barbe, Bâtiment Réaumur L5.02.02, B-1348, Louvain-la-Neuve, Belgium \\ ${ }^{2}$ A\&M Department, Metallic Materials Science Unit, Faculty of Applied Science, Université \\ de Liège, Quartier Polytech 1, 13A Allée de la Découverte, B-4000 Liège, Belgium \\ ${ }^{3}$ Department of Materials Science and Engineering, Monash University, 22 Alliance Lane, \\ Clayton, VIC 3800, Australia. \\ * Corresponding author. E-mail: aude.simar@uclouvain.be
}

\begin{abstract}
Friction Stir Processing (FSP) is an attractive manufacturing technique to produce Mg matrix composites since it avoids the problem of excessive reactivity between reinforcement and matrix encountered in liquid-phase processing routes. However, the strength of the interface in C-reinforced Mg matrix composites produced by FSP remains to be assessed. A short fibre composite has been fabricated by FSP a stack of a C-fabric between two Mg-AZ91D alloy sheets. In order to elucidate the interplay between matrix hardness and interface bonding strength, the work investigates the influence of heat treatment on the mechanical properties of the composites. An incremental Mori-Tanaka model is developed to analyse the relative roles of heat treatment and C-fibre reinforcement on the flow strength and ductility of the composites in tension and compression. The mean-field model provides an estimate of the stress at the matrix/fibre interface, from which a simple debonding criterion can be derived. Comparison
\end{abstract}


between model predictions and experimental data indicates that damage in the FSP composites is triggered by early interfacial debonding. Based on Finite Element simulations of a tensile test carried out in-situ in a scanning electron microscope, the critical interfacial stress for debonding was identified to be $435 \mathrm{MPa}$ in simple traction but only $250 \mathrm{MPa}$ when damage is governed by shear. This explains the limited strengthening by $\mathrm{C}$ fibres observed in heat treated composites.

Keywords: Magnesium Matrix Composites; Friction Stir Processing; Interface debonding; Micromechanics; Micromechanical modelling.

\section{Introduction}

Magnesium Matrix Composites (MgMCs) are attractive materials for structural applications requiring a combination of low weight and high strength. MgMCs take advantage of the intrinsically low density and high strength of Magnesium alloys, compared to traditional structural alloys like Aluminium. The presence of reinforcement further increases the stiffness and strength of the composite [1], while addressing some of the limitations of magnesium, such as poor creep resistance [2]. The mechanical properties of metal matrix composites are not only dominated by their fundamental constituents' properties but are highly affected by the interfacial bonding between their constituents. Interface debonding (also called interface decohesion) is the major damage mechanism in metal matrix composites [3].

Conventional molten metal routes for the production of MMC's, as e.g. squeeze casting or stir casting, are faced with important issues related to the matrix/reinforcement interface. On one hand, for $\mathrm{C}$ fibre MgMCs, the poor wettability between carbon and pure $\mathrm{Mg}$ [4-6] may result in inadequate distribution of the reinforcing phase in the metallic matrix [2,7]. On the other hand, excessive interfacial reactions between the reinforcement and the molten metal may prove detrimental to the integrity and mechanical behaviour of the composite. These poor mechanical 
properties are due to a significant degradation of the reinforcement and/or to the formation of brittle intermetallic compounds. In particular, extensive undesirable reactions have been reported between carbon and $\mathrm{Mg}$ alloys with a high $\mathrm{Al}$ content (such as alloy AZ91D) in the liquid-state and for high processing temperature $[4,6,8]$. Poor interface bonding may lead to premature failure by matrix decohesion from the $\mathrm{C}$ fibre [9].

Friction Stir Processing (FSP) is an alternative solid-state processing technology for the production of MgMCs that could potentially address the above-mentioned limitations [10]. Indeed, FSP has proved as a powerful technique to obtain a homogeneous distribution of reinforcing phase [11] particularly in the case of a limited liquid-state wetting of the reinforcement $[7,12]$. In addition, thanks to the relatively low processing temperature and short processing time in FSP, undesirable interfacial reactions may be avoided [12,13]. However, interface debonding is still the damage mechanism leading to the failure of the $\mathrm{C}$ fibre reinforced magnesium matrix composites processed by FSP [14]. Indeed, it remains the weakest interface in the material. No in-depth quantification of the interfacial debonding has been reported in literature for metal matrix composites processed by FSP.

Interface debonding in C fibre reinforced MgMCs has mainly been observed post-mortem on fractographies $[14,15]$. These observations do not allow a quantification of the strength of the interface. Few authors have attempted to quantify damage evolution in C fibre reinforced magnesium metal matrix composites $[9,16]$. Ju et al. [16] monitored the change in Young's modulus or anelastic residual strain and related these to the accumulated damage in their composite. Qi et al. [9] monitored the residual strength of the composite after various fatigue cycles and for various loads. These techniques remain global and allow providing a rate of damage accumulation. However, they do not allow to relate the measurements to the local stress state at the interface. 
In this work, we investigate the deformation under tension and compression and the damage behaviour of short fibre-reinforced composites produced by FSP through a combination of experiments and micromechanical modelling. The AZ91D (Mg-Al-Zn) alloy is used as matrix material, and the FSP processing route follows previous work [5,17]. Our technology presents significant advantages in terms of ease of implementation and scalability. It consists in applying FSP to an assembly made of a layer of $\mathrm{C}$ fabric placed between two sheets of $\mathrm{Mg}$ alloy (Figure 1). The microstructure obtained with this technique was investigated in a previous publication [14]. However, mechanical properties and damage mechanisms were not quantified. A tensile test is conducted on a notched specimen inside a scanning electron microscope (SEM) in order to observe in-situ the onset of interface debonding.

The respective roles of reinforcement and heat treatment are analysed by means of a micromechanical mean-field model. An incremental Mori-Tanaka model [18] is developed to predict the composite response based on the behaviour of individual phases. The model provides an estimate of the stress in the fibres, which is used to predict the onset of damage in tension and compression. Damage prediction relies on a simple debonding criterion which is calibrated on the basis of the in-situ notched tensile test. The micromechanical model suggests that damage in tension is governed by interfacial normal stress, while interfacial shear stress plays a key role in debonding under compression.

The paper is organised as follows. The experimental methods and experimental results are presented in Sections 2 and 3, respectively. The micromechanical model is described in Section 4 and 5. The experimental data and model predictions are discussed in view of the deformation and damage mechanisms in Section 6. 


\section{Experimental methods}

\subsection{Background: processing method}

The short C fibre-reinforced $\mathrm{Mg}$ composites considered in this study were obtained following the processing route presented in previous work [14]. The processing route and main processing parameters are thus only briefly recalled.

The matrix is commercial alloy AZ91D and was used in the form of $1.5 \mathrm{~mm}$ as-cast sheets. Samples for FSP were prepared by stacking one layer of C fabric (with mean fibre diameter of $7 \mu \mathrm{m}$ ) between two sheets of AZ91D alloy (Figure 1). The total thickness of the sandwich was $3.5 \mathrm{~mm}$. Reference samples made of two AZ91 layers without the $\mathrm{C}$ fabric were also processed by FSP. These will later be referred to as the unreinforced material. FSP of all the samples was performed under displacement control using a Hermle milling machine. The FSP parameters were chosen to ensure a uniform volume fraction of the reinforcement in the nugget zone, according to a previous study [17]. The advancing speed was set to $80 \mathrm{~mm} / \mathrm{min}$ and the rotational speed was set to $500 \mathrm{rpm}$ [14]. The nugget zone was found to be $10 \mathrm{~mm}$ wide and 1.5 mm thick.

The microstructures of the FSP materials were extensively characterised using optical microscopy, SEM and X-ray microtomography in Mertens et al. [14]. Based on detailed SEM characterisation, the volume fraction of C-fibre in the composite was found to be $7.1 \%$. A bimodal size distribution was revealed. Longer fibres have $6.5 \mu \mathrm{m}$-diameter and $15.6 \mu \mathrm{m}$ length. The shorter equi-axed fragments have a mean size of $1.5 \mu \mathrm{m}$. The small aspect ratio of 2.4 of the "longer" fibres justifies the assumptions of spherical reinforcements that will be made in the mean-field model. It was also found that, the grain size is slightly smaller in the composite $(6.2 \pm 1.9 \mu \mathrm{m})$ than in the unreinforced matrix $(9.3 \pm 3.1 \mu \mathrm{m})$. The fibres are homogenously distributed in the nugget zone (Figure 1, bottom). 
For the present work, half of the samples (both with and without $\mathrm{C}$ fibres) were subjected to a heat treatment $(\mathrm{HT})$ at $200^{\circ} \mathrm{C}$ for 8 hours after FSP.

\subsection{Mechanical testing methods}

Uniaxial tensile and compression tests were performed on specimens machined from the nugget zone of the FSPed materials with the loading direction in the processing direction. The flat specimens for tensile testing were $0.8 \mathrm{~mm}$ thick, $5 \mathrm{~mm}$ wide and $60 \mathrm{~mm}$ long, with an initial gauge length of $22 \mathrm{~mm}$. The specimens were loaded at a rate of $1 \mathrm{~mm} / \mathrm{min}$. Compression tests were performed on $1 \mathrm{~mm}$ diameter and $1.9 \mathrm{~mm}$ long cylindrical specimens at a rate of 1 $\mathrm{mm} / \mathrm{min}$. At least two tensile tests and four compression tests were performed for each material to assess reproducibility.

In order to investigate damage mechanisms in the composites, an in-situ tensile test was carried out in the SEM on a notched specimen of a composite after HT. The specimen was 0.8-mm thick, 5-mm wide, 25-mm long and presented a notch with 1-mm radius at mid-length: the reduced section was thus 3-mm wide. The specimen was submitted to standard polishing before testing. Displacements during tensile testing were measured between holding grips, with initial distance of $12 \mathrm{~mm}$. The tensile test was regularly interrupted to allow for a careful observation of the specimen in order to detect the onset of debonding between the fibres and the matrix. The forces at the onset of interfacial debonding and at final fracture were recorded.

Indentation tests allowed probing the behaviour of the matrix with and without $\mathrm{C}$ fibres. These tests were performed on the transverse section in the centre of the nugget zone using a Keysight Nano Indenter G200. The load was recorded as a function of indentation depth. A Berkovich indenter penetrated in the material up $1 \mu \mathrm{m}$, corresponding to about $30 \mathrm{mN}$ maximum load. For C fibre containing samples, small indents were positioned in between two regions presenting larger fibres (Figure 2). Small fragments of fibre were sometimes present inside the indents, as 
visible by the red circled fragments in the zoom in Figure 2. For unreinforced samples, a matrix of $3 \times 3$ indents was printed. The hardness was extracted from the recorded force vs indentation depth curve following the method of Oliver and Pharr [19]. The fit was performed at larger indentation depth (i.e. larger than $800 \mathrm{~nm}$ ) to avoid indentation size effects due to surface contaminants (such as oxides), surface work-hardened layer caused by sample preparation, surface roughness and indenter tip blunting [20].

\section{Experimental results}

\subsection{Effect of heat treatment on the matrix microstructure}

Figure 3 shows SEM micrographs after Nital etching of the reference (unreinforced) sample revealing the precipitation occurring after HT. Note that the grain size just after FSP has been characterized previously as described in section 2.1, see Mertens et al. [14] Fig. 9. No precipitates were observed just after FSP.

After HT, the microstructure of the unreinforced material (Figure 3) exhibits a high proportion of discontinuous precipitates, presenting a coarse lamellar morphology and whose formation was initiated at grain boundaries. These precipitates are $\mathrm{Mg}_{17} \mathrm{Al}_{12}$ intermetallics and have been largely documented in the literature of AZ91 [21-24]. No difference in matrix precipitation microstructure was observable in the $\mathrm{C}$ fibre containing samples.

\subsection{Matrix hardness}

Table 1 presents the matrix hardness probed by nanoindentation for the four types of samples. Small standard deviations attest for the reproducibility of the tests. The heat treatment causes a significant increase of matrix hardness, which is due to the precipitation observed in Figure 3. In samples without heat treatment, the matrix hardness increases significantly when carbon fibres are present. In contrast, the same increase is not observed in heat-treated samples. The 
increased matrix hardness when $\mathrm{C}$ fibres are present for samples without a heat treatment cannot be due to the presence of the fibres fragments observed on Figure 2 since no difference in matrix hardness due to the presence of the $\mathrm{C}$ fibres is observed after heat treatment. This fact also shows that precipitation (Figure 3) is not affected by the presence of $\mathrm{C}$ fibres. Hence, this difference appears to be due to a change of matrix deformation mechanisms after heat treatment, as will be discussed in section 6 .

\subsection{Flow properties in tension and compression}

Figure 4 shows the true stress-strain curve of the samples in uniaxial tension. The corresponding initial yield stress and fracture strain and stress are reported in Table 2 together with their standard deviation. For the materials without heat treatment, the presence of fibres significantly increases the flow stress, with an increase of $22 \mathrm{MPa}$ on the initial yield stress. This effect is also observed in the samples after heat treatment, although the increase of $6 \mathrm{MPa}$ in initial yield stress is comparatively lower. In both cases, the presence of fibres considerably reduces the fracture strain. On the other hand, the heat treatment increases the initial yield stress of the unreinforced material by $85 \mathrm{MPa}$. The tensile curve of the unreinforced material that was not submitted to a heat treatment presents a slight S-shape that has been attributed in literature to plastic deformation driven by twinning [25,26]. All other curves present a monotonously decreasing slope without evidence of such a S-shape.

True stress-strain curves in uniaxial compression are shown in Figure 5. Plastic and fracture properties in compression are also reported in Table 2. Compared to uniaxial tension tests, a larger variability is observed, which is expectedly due to the very small size of compression samples. Regarding the unreinforced materials without and with HT, one observes an asymmetry in the initial yield stress, with a 39 and $54 \mathrm{MPa}$ increase in initial yield stress in 
compression compared to tension. This difference is well known in AZ magnesium alloys and is qualified as the yield asymmetry, see e.g. Ref. [27-29]. The presence of fibres produces a 42 MPa increase in initial yield stress in the sample without HT, but no effect in the sample after HT (Table 2). Also, compared to tension tests, the influence of fibres on the fracture strain is generally much less significant. HT leads to an increase by $60 \mathrm{MPa}$ of the unreinforced material yield strength, which is slightly lower than the strengthening effect observed under tension.

Figure 6 shows post-failure SEM images taken at mid-thickness of tensile and compression samples without HT. These images where taken a few millimetres below the fracture surface. Similar observations were made for the samples after HT. For both tensile and compression specimens, there is a clear evidence of fibre-matrix debonding, and no fibre fracture could be observed. For the tensile specimens (Figure 6a), debonding is generally observed around the larger fibres, and takes place preferentially on particle surfaces that are oriented (roughly) perpendicularly to the tension direction. For the compression samples (Figure 6b), debonding is generally observed on interfaces that are parallel to the loading axis.

\subsection{In-situ notched tensile testing}

An in-situ tensile test was performed on a notched specimen of the composite after HT. The engineering stress in the notch is defined as the total force applied to the sample divided by the initial reduced section area at the notch of $3 \mathrm{~mm}$ width and $0.8 \mathrm{~mm}$ thickness. The engineering stress corresponding to the first observed debonding events is $280 \mathrm{MPa}$, while fracture occurs at an engineering stress in the notch of $320 \mathrm{MPa}$. Figure 7 shows SEM images in the notch area at the loading step when the onset of damage was observed. These images show damage nucleation by interfacial debonding (Figure 7a) and the early stages of void coalescence (Figure 7b). 


\section{Mean-field model}

A micromechanical analysis was carried out in order to elucidate the respective roles of heat treatment and addition of fibres on strengthening. The analysis relies on an incremental MoriTanaka model [30,31], according to which the composite response is predicted based on the properties of the constitutive phases and a description of the microstructure in terms of volume fraction and shape of the reinforcing phase. Given the bi-modal distribution of fibre shapes, including short fibres with relatively small aspect ratio of 2.4 as well as smaller fragments, the effect of fibre shape was neglected in the model and we simply assume spherical particles.

The model provides estimates of the effective response, as well as per-phase averages of the stress and strain fields. The predicted average stress in the fibres will be further exploited to predict the onset of interface debonding in Section 5.

\subsection{Constitutive models for the fibre and matrix phases}

The micromechanical model relies on a phenomenological description of the intrinsic behaviour of the phases. The behaviour of the $\mathrm{C}$ fibres is assumed to be isotropic and linear elastic, with Young modulus $E^{f}=230 \mathrm{GPa}$ and Poisson's ratio $v^{f}=0.2$. The matrix constitutive response is described by classical $\mathbf{J}_{2}$ elasto-plasticity theory with isotropic hardening. The Young modulus and Poisson's ratio were set to $E^{m}=45 \mathrm{GPa}$ and $v^{m}=0.3$, respectively. The initial yield stress and hardening parameters were fitted on representative experimental stress-strain curves corresponding to the unreinforced matrix, both with and without heat treatment (Figure 4 and 5). Different hardening models were adopted to describe the tension and compression flow response to account for the tension-compression asymmetry of the matrix behaviour. 
As mentioned in Section 3.3, the tensile plastic response of the unreinforced matrix without HT exhibits a S-shape (Figure 4). Fitting of the hardening behaviour required thus a 3 -stage, exponential-linear-exponential model:

$$
\begin{gathered}
\sigma_{Y}^{(1)}(p)=\sigma_{Y 0}+k_{1}\left[1-\exp \left(-n_{1} p\right)\right]\left(p \leq p_{1}\right) \\
\sigma_{Y}^{(2)}=\sigma_{1}+k_{2}\left(p-p_{1}\right) \quad\left(p_{1} \leq p \leq p_{2}\right) \\
\sigma_{Y}^{(3)}(p)=\sigma_{2}+k_{3}\left[1-\exp \left(-n_{3}\left(p-p_{2}\right)\right)\right] \quad\left(p_{2} \geq p\right)
\end{gathered}
$$

where $p$ is the accumulated plastic strain. The identified fitting parameters are the following: $\sigma_{Y 0}=80 \mathrm{MPa}, n_{1}=294, \sigma_{1}=172 \mathrm{MPa}, k_{2}=1818 \mathrm{MPa}, \sigma_{2}=280 \mathrm{MPa}$, and $n_{3}=9.7$ The other four parameters $k_{1}, k_{3}, p_{1}$ and $p_{2}$ were calculated to ensure continuity of the flow curve, $\sigma_{Y}^{(1)}\left(p_{1}\right)=\sigma_{Y}^{(2)}\left(p_{1}\right)$ and $\sigma_{Y}^{(2)}\left(p_{2}\right)=\sigma_{Y}^{(3)}\left(p_{2}\right)$, as well as continuity of its first derivative: $\sigma_{Y}^{(1) \prime}\left(p_{1}\right)=\sigma_{Y}^{(2) \prime}\left(p_{1}\right)$ and $\sigma_{Y}^{(2) \prime}\left(p_{2}\right)=\sigma_{Y}^{(3) \prime}\left(p_{2}\right)$. In contrast, the tensile plastic response of the matrix after HT could be fitted using a simple exponential (Voce) law, indicating a change in the dominant flow mechanism:

$$
\sigma_{Y}(p)=\sigma_{Y 0}+k[1-\exp (-n p)]
$$

with $\sigma_{Y 0}=235 \mathrm{MPa}, k=240 \mathrm{MPa}$ and $n=9.9$. The matrix responses with and without $\mathrm{HT}$, as modelled by $\mathrm{J} 2$ plasticity theory, are represented in Figure 8 a together with representative experimental curves (see light blue and orange curves).

The compressive matrix response (both with and without HT) was fitted with an exponential law of the form (4). The following set of parameters was identified: $\sigma_{Y 0}=202 \mathrm{MPa}, k=347$ $\mathrm{MPa}$ and $n=6.1$ (without HT), and $\sigma_{Y 0}=262 \mathrm{MPa}, k=331 \mathrm{MPa}$ and $n=7.4$ (with HT). The curve fitting for the matrix behaviour of the compression samples has been performed 
similarly to the tensile samples but only on the first $10 \%$ of plastic deformation in order to avoid the effect of barrelling on the specimen on the apparent flow properties (little barrelling has been observed in the specimens before failure). The result of the fit of the matrix response in compression is shown in Figure $8 \mathrm{~b}$ (see light blue and orange curves).

\subsection{Incremental Mori-Tanaka model}

The composite response was predicted based on the constitutive response of individual phases and on fibre volume fraction using a semi-analytical, nonlinear mean-field model. We assume that the behaviour of the matrix is unaffected by the addition of fibres, so that the constitutive models identified in the previous section can be used as an input for the mean-field model. This assumption will be discussed in Section 5 .

The two-phase composite is modelled using the Mori-Tanaka model [30,31]. In this model, the reinforcing particles are collectively represented as an isolated inhomogeneity embedded in an infinite medium and have the properties of the matrix. The particles are subjected to the matrix average strain as far-field strain. They are represented as having a spherical shape, conferring to the composite an isotropic effective response. The original Mori-Tanaka scheme was initially developed for linear elastic materials, in which case the isolated inclusion problem has an analytical solution [32]. In order to account for the elasto-plastic behaviour of the matrix, we adopt an incremental version of the Mori-Tanaka model as proposed by Doghri and Ouaar [18]. In that version of the model, the matrix response is linearised at each time step using the tangent operator of the matrix evaluated at the current matrix average strain. Specifically, a particular isotropic projection of the algorithmic tangent operator of $\mathrm{J} 2$ plasticity is used, see [18] for details. The incremental Mori-Tanaka model is outlined below.

At a given simulation time step, the average strain increment in the reinforcing phase, $\Delta \boldsymbol{\varepsilon}^{f}$, is linearly related to the matrix average strain increment $\Delta \varepsilon^{m}$ by the Mori-Tanaka relation: 


$$
\Delta \boldsymbol{\varepsilon}^{f}=\left[\boldsymbol{I}+\boldsymbol{P}:\left(\boldsymbol{C}^{f}-\boldsymbol{C}^{m}\right)\right]^{-1}: \Delta \boldsymbol{\varepsilon}^{m}
$$

where $\boldsymbol{C}^{f}$ and $\boldsymbol{C}^{m}$ are the $4^{\text {th }}$ order tangent operators of the fibre and the matrix materials, $\boldsymbol{I}$ is the $4^{\text {th }}$ order symmetric identity tensor, and $\boldsymbol{P}$ is Hill's polarisation tensor (related to Eshelby's tensor). $\boldsymbol{P}$ is calculated for the matrix tangent operator and the equivalent inclusion shape (a sphere). Expressions for $\boldsymbol{P}$ can be found for example in [33]. The per-phase average strain increments in Equation (5) are related to the (given) macroscopic strain increment $\Delta \overline{\boldsymbol{\varepsilon}}$ by the averaging condition:

$$
\Delta \overline{\boldsymbol{\varepsilon}}=v^{f} \Delta \boldsymbol{\varepsilon}^{f}+\left(1-v^{f}\right) \Delta \boldsymbol{\varepsilon}^{m}
$$

where $v^{f}$ is the fibre volume fraction, set to the experimental value, i.e. $v^{f}=7.1 \%$. Note that Equation (5) is nonlinear (through the strain dependence of the matrix tangent operator) and further requires the integration of the $\mathrm{J} 2$ plasticity equations at each time step. In practice, Equations (5)-(6) are solved simultaneously using a fully implicit scheme, and the J2 plasticity equations are integrated in time using the conventional radial return scheme.

After determination of average strain increments, the corresponding average stress increments in each phase are calculated from the constitutive relations of the phase, and the macroscopic stress increment is obtained by averaging:

$$
\Delta \overline{\boldsymbol{\sigma}}=v^{f} \Delta \boldsymbol{\sigma}^{f}+\left(1-v^{f}\right) \Delta \boldsymbol{\sigma}^{m}
$$

The incremental Mori-Tanaka model was used to predict the composite response without and with HT, in uniaxial tension and compression. The comparison between model predictions and experimental data is shown in Figure 8. Overall, the Mori-Tanaka model well captures the yield strength and hardening rate of the composite. A notable exception is the compressive response 
of the composite after heat treatment: in this case the composite effect predicted by the model is larger than the experimental one.

Also note that the model is often inaccurate during the elasto-plastic transition. The MoriTanaka model predicts a sharp elasto-plastic transition, similar to the elasto-plastic transition in the constitutive model of the matrix (also shown in Figure 8). In contrast, experimental curves exhibit a smoother transition, which is attributed to stress and strain inhomogeneities within the matrix. This limitation is intrinsic to the adopted model, which describes the matrix response based on a single, average measure of strain.

\subsection{Numerical simulations of tensile testing of a notched sample}

The incremental Mori-Tanaka model was used to simulate the tensile testing of a notched sample of the composite after HT. Since macroscopically heterogeneous fields develop in the sample due to the presence of the notch, a FE simulation was carried out using the commercial software Abaqus [34]. At every integration point of the FE mesh, the macroscopic stress-strain response of the composite was calculated using the incremental Mori-Tanaka model. This was achieved by implementing the model as a user subroutine (UMAT). Note that this required the implementation of the algorithmic tangent operator corresponding to the fully-implicit timeintegration of the mean-field model. The properties of the individual phases used as an input of the Mori-Tanaka model are identical to the properties used to simulate tensile tests of the composite after HT (Fig. 8a). Thus, the matrix behaviour used in these simulations is taken from the fit of the tensile curve for the unreinforced sample after HT, as described in Section 4.1.

Figure 9 shows the mesh used for the FE simulation. Symmetry of geometry and loading conditions in the longitudinal and transverse directions were taken into account, and only $1 / 4$ of the actual sample geometry was meshed. Symmetry boundary conditions were applied on 
the inner surfaces, and imposed displacement boundary conditions were applied to the top surface. The "strip" region indicated on Figure 9 corresponds to the sample region where the in-situ SEM observations were made. Microscopic fields useful for the investigation of damage mechanisms were extracted from integration points in the strip region. An example of deformed configuration is shown on the right-hand side of Figure 9 to illustrate the heterogeneous fields that develop in a notched sample. The colour map shows the field of macroscopic stress $\bar{\sigma}_{11}$ in the direction of applied tension, at the deformation step corresponding to the onset of debonding as identified from the SEM observations in Figure 7a (see below).

Figure 10a shows the engineering stress in the notch (previously defined as the total applied force on the top of the sample, divided by the reduced area at the notch) as a function of the normalised applied displacement $u / L$, with $L$ the sample half-length ( $L=6 \mathrm{~mm}$ ). The numerical predictions reproduce the experimental curve well without any additional fitting. The horizontal dashed line indicates the onset of interface debonding based on SEM observation, namely when the engineering stress in the notch reaches $280 \mathrm{MPa}$. The deformation step corresponding to this level of stress is then identified in the FE model.

During loading, the field of fibre average stress $\boldsymbol{\sigma}^{f}$ predicted by the Mori-Tanaka model is heterogeneous in the simulation volume. In order to obtain a representative value of the fibre stress at the onset of debonding near the notch, a volume average of the fibre average stress field $\boldsymbol{\sigma}^{f}$ was carried out over the strip volume: $\boldsymbol{\sigma}_{(\text {in notch })}^{f}=\left(\sum V_{i} \boldsymbol{\sigma}_{i}^{f}\right) / \sum V_{i}$, where $V_{i}$ is the volume of the $\mathrm{i}^{\text {th }}$ integration point in the strip region, and $\boldsymbol{\sigma}^{f}{ }_{i}$ the fibre average stress at that integration point. The same procedure was used to obtain a representative value of the macroscopic stress near the notch, $\overline{\boldsymbol{\sigma}}_{(\text {in notch })}^{f}$ The evolution of the macroscopic and fibre average stress in the strip region, $\bar{\sigma}_{11,(i n \text { notch })}$ and $\sigma_{11,(\text { in notch })}^{f}$, are shown in Figure $10 \mathrm{~b}$ as a function of the normalised applied displacement. At the deformation step corresponding to the 
onset of debonding, the fibre stress $\sigma_{11,(\text { in notch) }}^{f}$ was found to be $435 \mathrm{MPa}$. This value coincides with the maximum principal stress of the tensor $\boldsymbol{\sigma}_{(\text {in notch })}^{f}$. Note that, according to the MoriTanaka model, the mean fibre stress $\sigma_{11,(\text { in notch) }}^{f}$ is significantly larger than the mean macroscopic stress in the notch, $\bar{\sigma}_{11,(\text { in notch) }}$, which is slightly below $300 \mathrm{MPa}$ at the onset of debonding, as can be observed in Figure 9 and 10b. This is not surprising given the relatively large stiffness contrast between the fibre and matrix phases. In the next section, we use the simpler notation $\boldsymbol{\sigma}^{f}$ to indicate the mean fibre stress in the strip region, for conciseness.

\section{Prediction of the onset of damage by interface debonding}

At each deformation step, the mean-field model relies on Eshelby's solution for a spherical representative inclusion in an infinite matrix (Figure 11a). Accordingly, the stress in this representative inclusion representing the $\mathrm{C}$ fibres is uniform. It can therefore be used to calculate the surface traction vector on the interface (force per area exerted by the matrix on the inclusion) using Cauchy's formula (accounting for the symmetry of the stress tensor): $\boldsymbol{t}(\boldsymbol{n})=$ $\boldsymbol{\sigma}^{f} \cdot \boldsymbol{n}$, where $\boldsymbol{n}$ is the local normal vector on the interface pointing towards the matrix side. By projection along the normal and tangential directions, the surface traction vector can be decomposed into its normal and tangential components:

$$
\sigma_{n}^{i n t}(\boldsymbol{n})=\boldsymbol{t}(\boldsymbol{n}) \cdot \boldsymbol{n}, \tau^{i n t}(\boldsymbol{n})=\sqrt{|\boldsymbol{t}(\boldsymbol{n})|^{2}-\left(\sigma_{n}^{i n t}\right)^{2}}
$$

The normal stress $\sigma_{n}^{\text {int }}$ on the interface can be positive or negative. The resultant shear stress $\tau^{\text {int }}$ is positive by definition (8).

We proceed by using these interfacial stress measures in a debonding criterion. A simple criterion assumes that debonding starts when the interfacial normal stress reaches a critical 
threshold (in tension), or when the resultant shear stress reaches a critical threshold, whichever happens first:

$$
\sigma_{n}^{i n t}(\boldsymbol{n})=\sigma_{c} \quad \text { or } \quad \tau^{i n t}(\boldsymbol{n})=\tau_{c}
$$

where $\sigma_{c}$ and $\tau_{c}$ are the critical interface traction and shear, respectively.

The interface parameters can be partially identified based on the results of the in-situ tensile tests in combination with the mean-field model. As explained in section 4.3, the FE simulations of the notched samples provides an estimate of the fibre average stress tensor at the onset of debonding, $\boldsymbol{\sigma}^{f}$. In particular, we found that $\sigma_{11}^{f}=435 \mathrm{MPa}$. From the full tensor $\boldsymbol{\sigma}^{f}$, the normal and resultant shear stresses at any point on the interface can be calculated using Equations (8). Because of the spherical symmetry of the representative inclusion in the model, it is sufficient to consider an arc of circle in the 1-2 plane, with normal vector $\boldsymbol{n}=$ $[\cos \alpha, \sin \alpha, 0], \alpha=\left[0,90^{\circ}\right]$ (Figure 11a). The evolutions under tensile loading of the normal and resultant shear stresses with the normal direction along the interface are shown in Figure 11b. The normal stress is highest $(435 \mathrm{MPa})$ at the pole in the tensile direction $\left(\alpha=0^{\circ}\right)$. The resultant shear stress reaches its maximum (180 MPa) for $\alpha=45^{\circ}$, and vanishes at the poles (the poles in the 1-2 directions are thus principal directions). The maximum shear stress is significantly lower than the maximum normal stress. Since debonding was predominantly observed at the poles in the tensile direction (Figure 7), we conclude that the normal stress criterion in Eq. (9) is reached first, and identify the critical stress: $\sigma_{c}=435 \mathrm{MPa}$. We further deduce that $\tau_{c}>180 \mathrm{MPa}$, or, equivalently, $\frac{\tau_{c}}{\sigma_{c}}>0.4$

We use the critical normal stress identified above to predict interface debonding in uniaxial tension. Similar to the previous analysis, the fibre average stress predicted by the mean-field model in uniaxial tension can be used to calculate the normal and resultant shear stress for all 
normal orientations. The evolution of the normal and resultant shear stress with the angle $\alpha$ at any stage of deformation is qualitatively similar to the evolution in the strip region of the notched sample, with a maximum normal stress reached at the pole in the tensile direction, and a maximum shear stress always lower than the maximum normal stress (Figure 11b). The predicted onset of debonding, according to the maximum normal stress criterion, is indicated with a black cross in Figure 8a. Here, we assumed that the heat treatment had no impact on the interface strength, and used the same criterion for the samples with and without heat treatment. The predicted onset of debonding is consistent with the experimental observations, with debonding occurring shortly before final fracture. The tensile strains at debonding predicted by the model are reported in Table 3. The uniaxial tension test also helps us refine the bound on the critical shear stress for debonding. At the onset of debonding, the maximum shear stress on the interface is $250 \mathrm{MPa}$, so that $\frac{\tau_{c}}{\sigma_{c}}>0.6$.

We repeat the same exercise for uniaxial compression. A representative profile of normal and resultant shear stress as a function of normal orientation is represented in Figure 11c. The maximum normal stress is tensile and occurs at the poles in the plane perpendicular to the compression direction. Here, the application of a maximum normal stress criterion (with the same previously identified critical normal stress) predicts the onset of debonding at excessively high values of compressive strains. This would be in contradiction with the experimental observations that show limited effect of the reinforcements on strength in compression, particularly after HT (Figure 5). It is thus necessary to invoke a shear-dominated mechanism. Considering a critical shear stress of $0.6 \sigma_{c}$ (corresponding to the lower bound on $\tau_{c}$ identified above), we can provide corresponding lower bounds on the onset of shear-driven debonding, which are represented by black crosses in Figure $8 \mathrm{~b}$. The compressive strains at debonding predicted by the model are also reported in Table 3. Experimentally, failure does not occur at 
interface debonding due to the re-filling of the generated void thanks to the plasticity of the matrix and the compressive loading.

\section{Discussion}

\subsection{Effect of heat treatment and yield asymmetry on the flow stress}

The heat treatment induces a strengthening of the matrix in both tension and compression (Figure 4 and 5). The strengthening can essentially be attributed to the presence of $\mathrm{Mg}_{17} \mathrm{Al}_{12}$ precipitates (Orowan strengthening [35]) (Figure 3) as largely reported in the literature for AZ91 alloys [21-24,35]. In addition, the heat treatment appears to bring about a change in the deformation mechanism in the unreinforced matrix under tension, as suggested by the S-shape of the tensile curve (Figure 4) leading to a change of hardening model chosen in Section 4.1. Indeed, fitting of the flow curves of the untreated matrix required a complex, 3-stage hardening model to capture the S-shape curve which reflects the occurrence of twinning $[25,26]$. In contrast, the flow curve of the heat-treated matrix is well-fitted by a simple Voce law, which suggests a slip-dominated behaviour. We attribute this transition to the presence of the $\mathrm{Mg}_{17} \mathrm{Al}_{12}$ precipitates, which causes a change in matrix behaviour from twinning-dominated behaviour (without HT) to slip-dominated behaviour (with HT) as known in magnesium alloys [27,28]. Jain et al. [27] have shown a significant decrease of the $\{10 \overline{1} 2\}$ extension twin volume fraction under straining in an aged condition of an $\mathrm{Mg}-8 \mathrm{Al}-0.5 \mathrm{Zn}$ alloy compared to the solution treated material. This effect is due to the difficulty for twin boundaries to migrate in a high density of precipitates [36].

The texture after FSP of magnesium alloys is well documented and shows a strong preferential orientation with a tendency for the $\mathrm{c}$ axis of the $\mathrm{HCP}$ lattice to align with the processing direction $[37,38]$, i.e. the basal plane tends to become perpendicular to our loading direction. Extension twinning are favoured in the case of a tensile stress perpendicular to the basal plane 
and a compressive stress parallel to the basal plane [27]. This justifies the favourable twinning in the tensile tests compared to the compression tests performed in our study. The polar nature of the extension twins of Mg causes thus yield asymmetry [27-29]. The compressive flow response is always slip dominated and well-captured by a Voce law. No change in mechanism seems to be induced by the heat treatment. The strengthening due to the heat treatment is thus attributed to the Orowan mechanism [35].

\subsection{Effect of $C$ fibres}

In uniaxial tension, a composite effect upon addition of carbon fibres is observed on all experimental curves (Figure 4). The increase in yield stress due to this composite effect is well captured by the Mori-Tanaka mean-field model (Figure 8). The model however does not accurately capture the shape of the hardening curve in the reinforced sample. Indeed, while the composite hardening curve predicted by the model still exhibits a S-shape stemming originating from the matrix constitutive model, the S-shape is not observed in the experimental curve of the composite sample without HT. We believe that this reflects the fact that the matrix flow mechanism undergoes a transition from twinning-dominated (unreinforced matrix) to slipdominated (reinforced matrix) deformation due to the grain size decrease associated to the fibres. Indeed, the grain size decreases from $9.3 \pm 3.1 \mu \mathrm{m}$ for the unreinforced samples to $6.2 \pm$ $1.9 \mu \mathrm{m}$ for the reinforced sample (Section 2.1) [14]. The effect of grain size on the transition from twinning-dominated to slip-dominated deformation mechanism in $\mathrm{Mg}$ is largely reported in literature [39-41]. Cepeda-Jiménez et al. [41] report a $60 \%$ twin fraction in a $19 \mu \mathrm{m}$ grain size pure $\mathrm{Mg}$ while only $14 \%$ is reported in their $5 \mu \mathrm{m}$ grain size sample. Chino et al. [40] report limited deformation by twining in AZ31 for a grain size of $8 \mu \mathrm{m}$, i.e. a grain size similar to our reinforced sample. 
The nanoindentation results confirm the modification of matrix behaviour for the non-heat treated samples when adding the $\mathrm{C}$ fibres. Indeed, a $0.08 \mathrm{GPa}$ increase in nanohardness of the matrix is found between the unreinforced material and the $\mathrm{C}$ fibre reinforced composite. Stresses under an indenter could potentially activate extension twinning which seems to be the case in our unreinforced and untreated sample. This indeed confirms that the matrix undergoes a significant intrinsic strengthening, which is induced by the presence of the fibres but is distinct from the composite effect. In comparison, nanoindentation tests in the matrix of the heat-treated samples indicated no significant strengthening upon addition of fibres within the range of experimental error. This indicates no modification in the deformation mechanism nor additional strengthening of any kind due to the addition of the $\mathrm{C}$ fibres in the heat-treated samples.

The presence of fibres significantly reduces the failure strain, as compared to the unreinforced material. This is attributed to premature damage triggered by matrix-fibre debonding. Debonding is followed by growth and coalescence of cavities leading to fast fracture in tension after debonding (Figure 8a and Table 2). The onset of debonding is reasonably well predicted by a simple criterion involving a critical stress identified from the in-situ tensile experiments. Earlier debonding is favoured in the HT samples due to matrix strengthening, as well predicted by the model, see Table 3. This can be directly correlated to the lower fracture strain of the HT composites (Figure 8a and Table 2).

Regarding the compression response, the amplitude of the composite effect observed for the samples without HT is underestimated by the mean-field model. In contrast, after heat treatment, the experimental composite effect is almost negligible, whereas the model overestimates the composite effect, especially at large strain. The negligible composite effect observed experimentally after HT could thus be attributed to early interfacial debonding dominated by shear, as suggested by the model (Table 3). In compression, the final fracture 
after onset of debonding is expected to be delayed, as the newly created voids are refilled by the matrix flow during further straining.

\section{Conclusion}

The deformation and damage behaviour of FSPed materials was investigated based on a nonlinear mean-field model. A simple debonding criterion was proposed, and the critical normal and shear stresses for debonding were estimated based on in-situ observations. Two strengthening effects were considered, namely the presence of C-fibres introduced via FSP, and the heat treatment performed after FSP. The main conclusions of this work are the following:

- Heat treatment induces a significant strengthening in both the unreinforced and the fibre-reinforced material. This can be attributed to Orowan strengthening arising from the growth of intermetallic precipitates. In addition, the change in strain hardening in the unreinforced matrix (as indicated by the fitting of the hardening law) and the nanoindentation results suggest a change in the flow mechanism of the matrix expectedly from twinning-dominated to slip-dominated.

- The presence of fibres induces a composite effect in both heat-treated and untreated samples, which is well captured by the mean-field model. The presence of fibres seems however to induce a change in the flow mechanism in the untreated material, which could be attributed to a decrease in grain size due to the presence of the fibres.

- The presence of fibres reduces the failure strain in tension as compared to the unreinforced FSPed alloy. Damage is attributed to the onset of interfacial debonding dominated by normal traction stress, which thus occurs earlier in high flow stress specimens. Early debonding dominated by shear stress could explain the reduction (or even the loss) of composite effect under compression in the heat-treated samples. 
- The combination of in-situ tensile testing and mean field modelling allowed to identify the debonding stress as equal to $435 \mathrm{MPa}$ in tensile tests, but equal to only $250 \mathrm{MPa}$ when damage is dominated by shear.

\section{Acknowledgements}

The authors acknowledge the financial support of the Interuniversity Attraction Poles Program from the Belgian State through the Belgian Policy agency, contract IAP7/21 INTEMATE. This research has also been funded (from January 2017) by the European Research Council (ERC) under the European Union's Horizon 2020 research and innovation program (grant agreement $\left.\mathrm{n}^{\circ} 716678\right)$.

\section{References}

[1] M. Gupta, N.M.L. Shanon, Magnesium, magnesium alloys, and magnesium composites. Hoboken, New Jersey, John Wiley and Sons, 2011.

[2] H.Z. Ye, X.Y. Liu, Review of recent studies in magnesium matrix composites, J. Mater. Sci. 39 (2004) 6153-6171.

[3] A. Mortensen, J. Llorca, Metal matrix composites, Annu. Rev. Mater. Res., 40 (2010) 243-270.

[4] J.C. Viala, G. Claveyrolas, F. Bosselet, J. Bouix, The chemical behaviour of carbon fibres in magnesium base Mg-Al alloys, J. Mater. Sci. 35 (2000) 1813-1825.

[5] A. Mertens, H.-M. Montrieux, J. Halleux, J. Lecomte-Beckers, F. Delannay, Processing of carbon fibers reinforced $\mathrm{Mg}$ matrix composites via pre-infiltration with Al, J. Mater. Eng. Perform. 21 (2012) 701-706.

[6] S. Li, L. Qi, T. Zhang, L. Ju, H. Li, Interfacial microstructure and mechanical properties of Cf/AZ91D composites with TiO2 and PyC fiber coatings, Micron 101 (2017) 170-176.

[7] P. Asadi, G. Faraji, A. Masoumi, M.K. Besharati-Givi, Experimental investigation of magnesium-base nanocomposite produced by friciton stir processing: effects of particle types and number of friction stir processing passes, Metall. Mater. Trans. A 42 (2011) 2820-2832.

[8] A. Olszowka-Myalska, Some physicochemical phenomena observed during fabrication of Mg-C cast composites, J. Mater. Eng. Perform. 25 (2016) 3091-3097.

[9] L. Qi, L. Ju, J. Zhou, S. Li, T. Zhang, W. Tian, Tensile and fatigue behavior of carbon fiber reinforced magnesium composite fabricated by liquid-solid extrusion following vacuum 
pressure infiltration, J. Alloys Comp. 721 (2017) 55-63.

[10] M.-N. Avettand-Fènoël, A. Simar, R. Shabadi, R. Taillard, B. de Meester, Characterization of oxide dispersion strengthened copper based materials developed by friction stir processing, Mater. Design 60 (2014) 343-357.

[11] Y. Morisada, H. Fujii, T. Nagaoka, M. Fukusumi, MWCNT/AZ31 surface composites fabricated by friction stir processing, Mater. Sc. Engin. A 419 (2006) 344-348.

[12] V. Sharma, U. Prakash, B.V. Manoj Kumar, Surface composites by friction stir processing: a review, J. Mater. Process. Technol. 224 (2015) 117-134.

[13] B. Ratna Sunil, G. Pradeep Kumar Reddy, H. Patle, R. Dumpala, Magnesium based surface metal matrix composites by friction stir processing, J. Magn. Alloys 4 (2016) 52-61.

[14] A. Mertens, A. Simar, J. Adrien, E.Maire, H.-M. Montrieux, F. Delannay, J. LecomteBeckers, Influence of fibre distribution and grain size on themechanical behavior of friction stir processed Mg-C composites, Mater. Charact. 107 (2015) 125-133.

[15] S. Zhang, G. Chen, R. Pei, M. Hussain, Y. Wang, D. Li, P. Wang, G. Wu, Effect of Gd content on interfacial microstructures and mechanical properties of $\mathrm{Cf} / \mathrm{Mg}$ composite, Mater. Design 65 (2015) 567-574.

[16] L. Ju, L. Qi, X. Wei, J. Zhou, X. Hou, H. Li, Damage mechanism and progressive failure analysis of Cf/Mg composite, Mater. Sci. Engin. A 666 (2016) 257-263.

[17] A. Mertens, A. Simar, H.-M. Montrieux, J. Halleux, F. Delannay, J. Lecomte-Beckers, Friction stir processing of magnesium matrix composites reinforced with carbon fibres: Influence of the matrix characteristics and of the processing parameters on microstructural developments, Proc. of the $9^{\text {th }}$ Int. Conf. Mg Alloys and their Applications, 2012, W.J. Poole and K.U. Kainer eds, pp. 845-850

[18] I. Doghri, A. Ouaar, Homogenization of two-phase elasto-plastic composite materials and structures: Study of tangent operators, cyclic plasticity and numerical algorithms, Int. J. Solids Struct. 40 (2003) 1681-1712.

[19] W.C. Oliver, G.M. Pharr, An improved technique for determining hardness and elastic modulus using load and displacement sensing indentation experiments, J. Mater. Research 7 (1992) 1564-1583.

[20] G.M. Pharr, E.G. Herbert, Y. Gao, The Indentation Size Effect: A Critical Examination of Experimental Observations and Mechanistic Interpretations, Annu. Rev. Mater. Res. 40 (2010) 271-292.

[21] S. Celotto, TEM study of the continuous precipitation in Mg-9\%Al-1\%Zn alloy, Acta Mater. 48 (2000) 1775-1787.

[22] Z.Y. Ma, Friction stir processing technology: a review, Metall. Mater. Trans. A 39 (2008) 642-658.

[23] W. Yuan, S.K. Panigrahi and R.S. Mishra, Achieving high strength and high ductility in friction-stir processed cast magnesium alloy, Metall. Mater. Trans. A 44 (2013) 3675- 3684. 
[24] V. Jain, R.S. Mishra, A.K. Gupta and Gouthama, Study of $\beta$-precipitates and their effect on the directional yield asymmetry of friction stir processed and aged AZ91C alloy, Mater. Sci. Eng. A 560 (2013) 500-509.

[25] S.R. Agnew, J.A. Horton, T.M. Lillo and D.W. Brown, Enhanced ductility in strongly textured magnesium produced by equal channel angular processing, Scr. Mater. 50 (2004) $377-381$.

[26] W. Yuan and R.S. Mishra,_Grain size and texture effects on deformation behavior of AZ31 magnesium alloy, Mater. Sci. Eng. A 558 (2012) 716-724.

[27] J. Jain, W.J. Poole, C.W. Sinclair, M.A. Gharghouri, Reducing the tension-compression yield asymmetry in a Mg-8Al-0.5Zn alloy via precipitation, Scripta Mater. 62 (2010) 301304.

[28] J.D. Robson, N. Stanford, M.R. Barnett, Effect of precipitate shape on slip and twinning in magnesium alloys, Acta Mater. 59 (2011) 1945-1956.

[29] C.M. Cepeda-Jimenez, M.T. Perez-Prado, Microplasticity-based rationalization of the room temperature yield asymmetry in conventional polycrystalline Mg alloys, Acta Mater. 108 (2016) 304-316.

[30] T. Mori, K. Tanaka, Average stress in matrix and average elastic energy of materials with misfitting inclusions, Acta Metall. 21 (1973) 571-574.

[31] Y. Benveniste, A new approach to the application of Mori-Tanaka's theory in composite materials. Mech. Mater. 6 (1987) 147-157.

[32] J.D. Eshelby, The determination of the elastic field of an ellipsoidal inclusion, and related problems. Proc. R. Soc. London A241 (1957) 376-396.

[33] L. Walpole, Elastic behavior of composite materials: theoretical foundations, Adv. Appl. Mech. 21 (1981) 169-203.

[34] ABAQUS/Standard, 2013. SIMULIA, Providence, RI.

[35] N. Stanford, J. Geng, Y.B. Chun, C.H.J. Davies, J.F. Nie, M.R. Barnett, Effect of plateshaped particle distributions on the deformation behaviour of magnesium alloy AZ91 in tension and compression, Acta Mater. 60 (2012) 218-228.

[36] M.A. Gharghouri, G.C. Weatherly, J.D. Embury, The interaction of twins and precipitates in a Mg-7.7 at.\% Al alloy, Phil. Mag. A 78 (1998) 1137-1149.

[37] W. Yuan, R.S. Mishra, B. Carlson, R.K. Mishra, R. Verma, R. Kubic, Effect of texture on the mechanical behavior of ultrafine grained magnesium alloy, Scripta Mater. 64 (2011) 580-583.

[38] M. Vargas, S. Lathabai, P.J. Uggowitzer, Y. Qi, D. Orlov, Y. Estrin, Microstructure, crystallographic texture and mechanical behaviour of friction stir processed $\mathrm{Mg}-\mathrm{Zn}-\mathrm{Ca}-\mathrm{Zr}$ alloy ZKX50, Mater. Sci. Engin. A 685 (2017) 253-264. 
[39] J. Koike, Enhanced deformation mechanisms by anisotropic plasticity in polycrystalline Mg alloys at room temperature, Metall. Mater. Trans. A, 36 (2005) 1689-1695.

[40] Y. Chino, K. Kimura, M. Mabuchi, Twinning behavior and deformation mechanisms of extruded AZ31 Mg alloy, Mater. Sci. Eng. A, 486 (2008) 481-488.

[41] C.M. Cepeda-Jimenez, J.M. Molina-Aldareguia, M.T. Perez-Prado, Origin of the twinning to slip transition with grain size refinement, with decreasing strain rate and with increasing temperature in magnesium, Acta Mater. 88 (2015) 232-244.

\section{Figures}

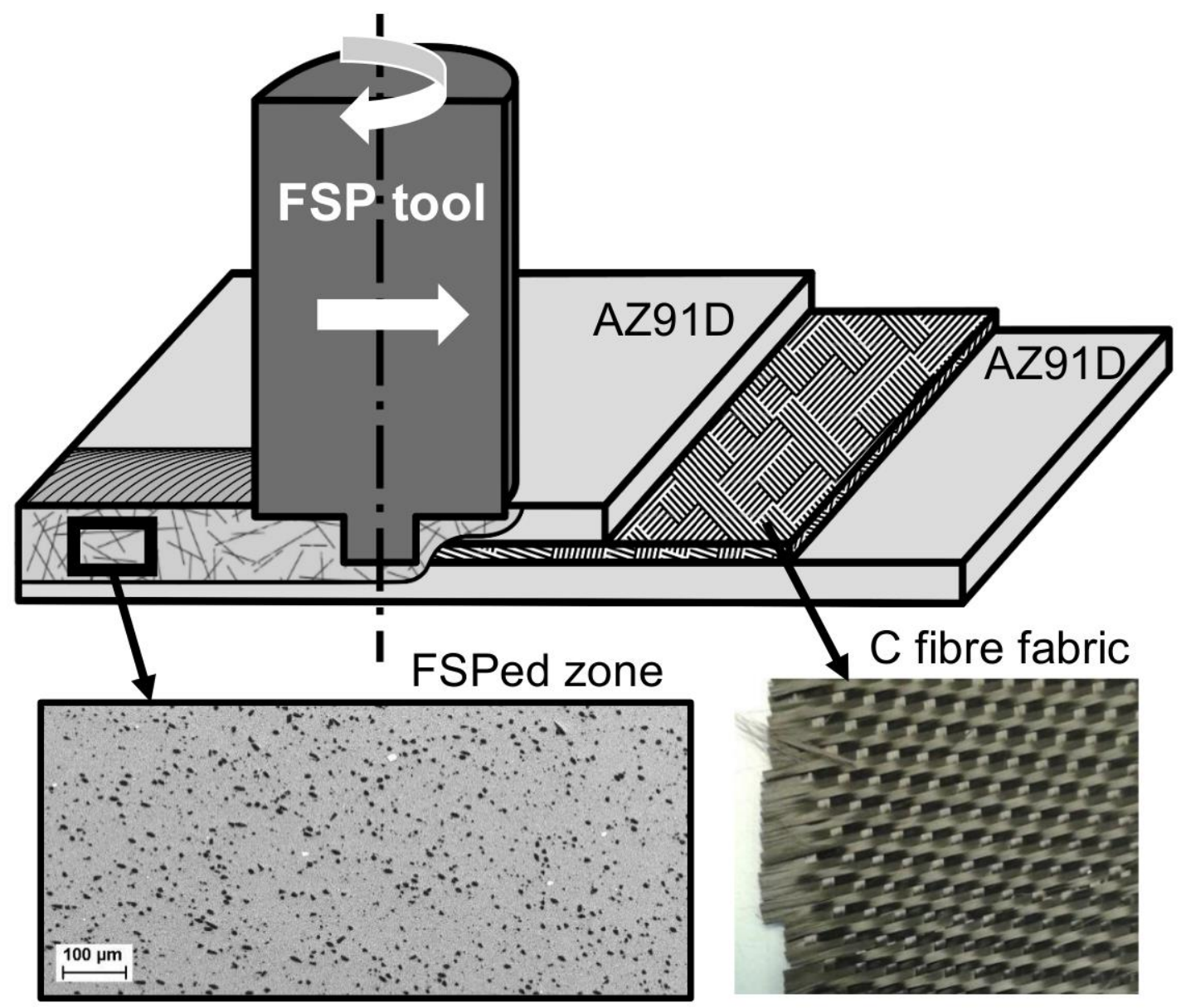

Figure 1. Schematic representation of the processing method used to integrate the carbon fibres in AZ91D alloy by FSP. Bottom: scanning electron micrograph of the processed zone after integration of the carbon fibres by FSP. 


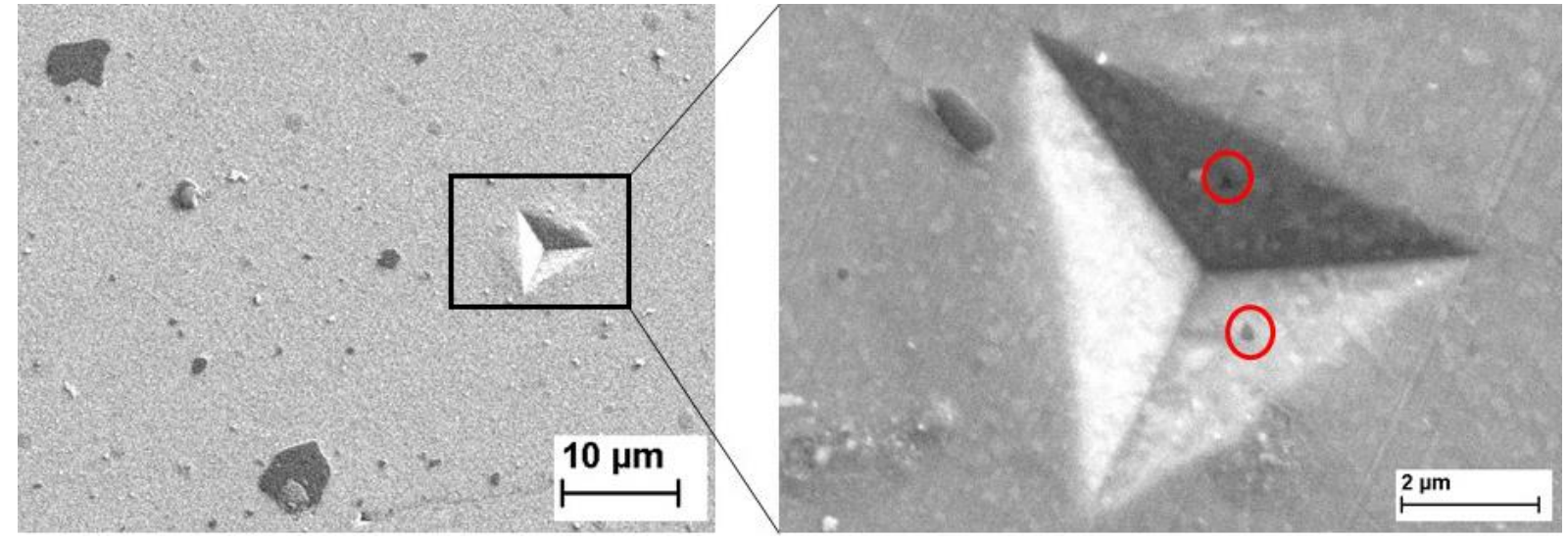

Figure 2. SEM micrograph of a Berkovich indent performed in the matrix of a C fiber FSPed sample with a maximum indentation depth of $1 \mu \mathrm{m}$. The red circles on the zoomed image (right) highlight the presence of sub-micron sized C fiber fragments. 


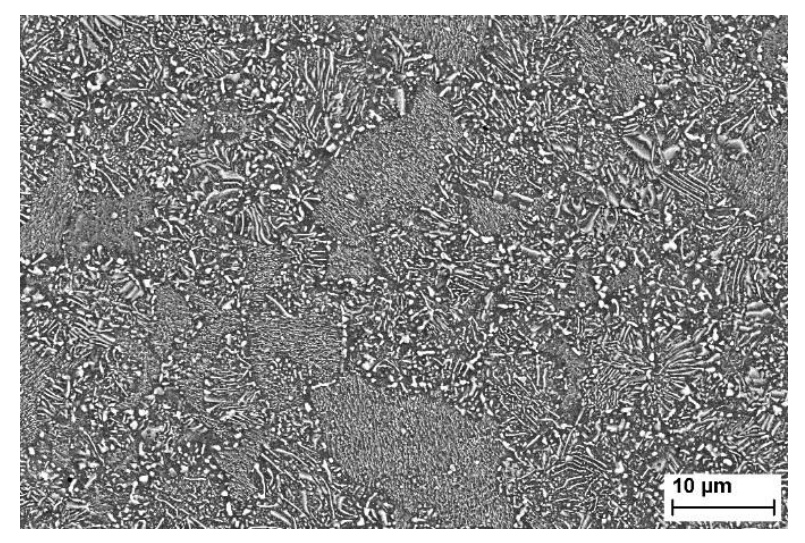

Figure 3. SEM micrographs of the unreinforced FSPed sample heat treated at $200^{\circ} \mathrm{C}$ for 8 hours. 


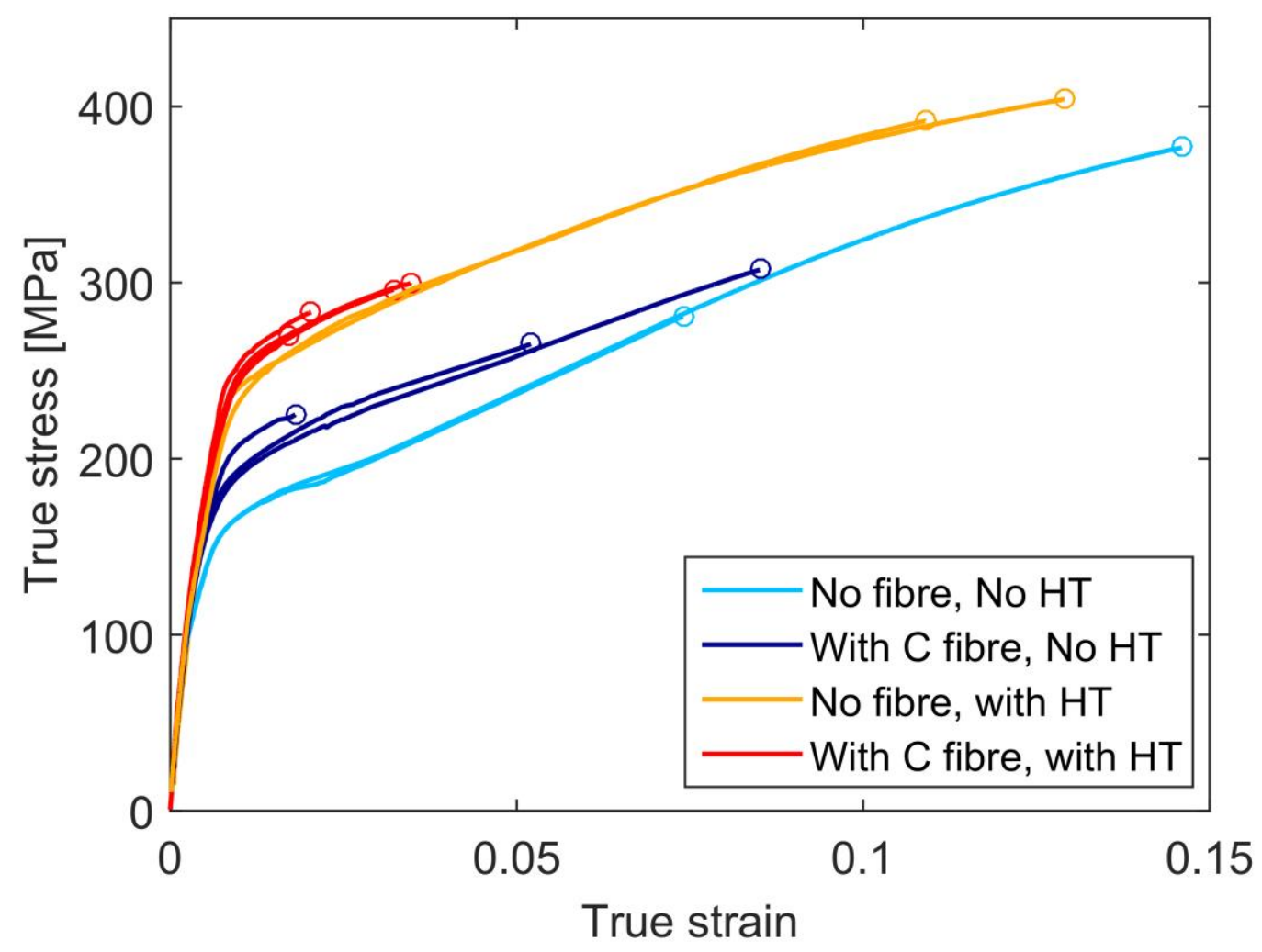

Figure 4. Experimental true stress-strain curves of the four FSP materials in uniaxial tension: Reference (unreinforced) and composite samples with and without heat treatment. The curves are represented up to the final fracture and the fracture stress and strain are represented by a circle. 


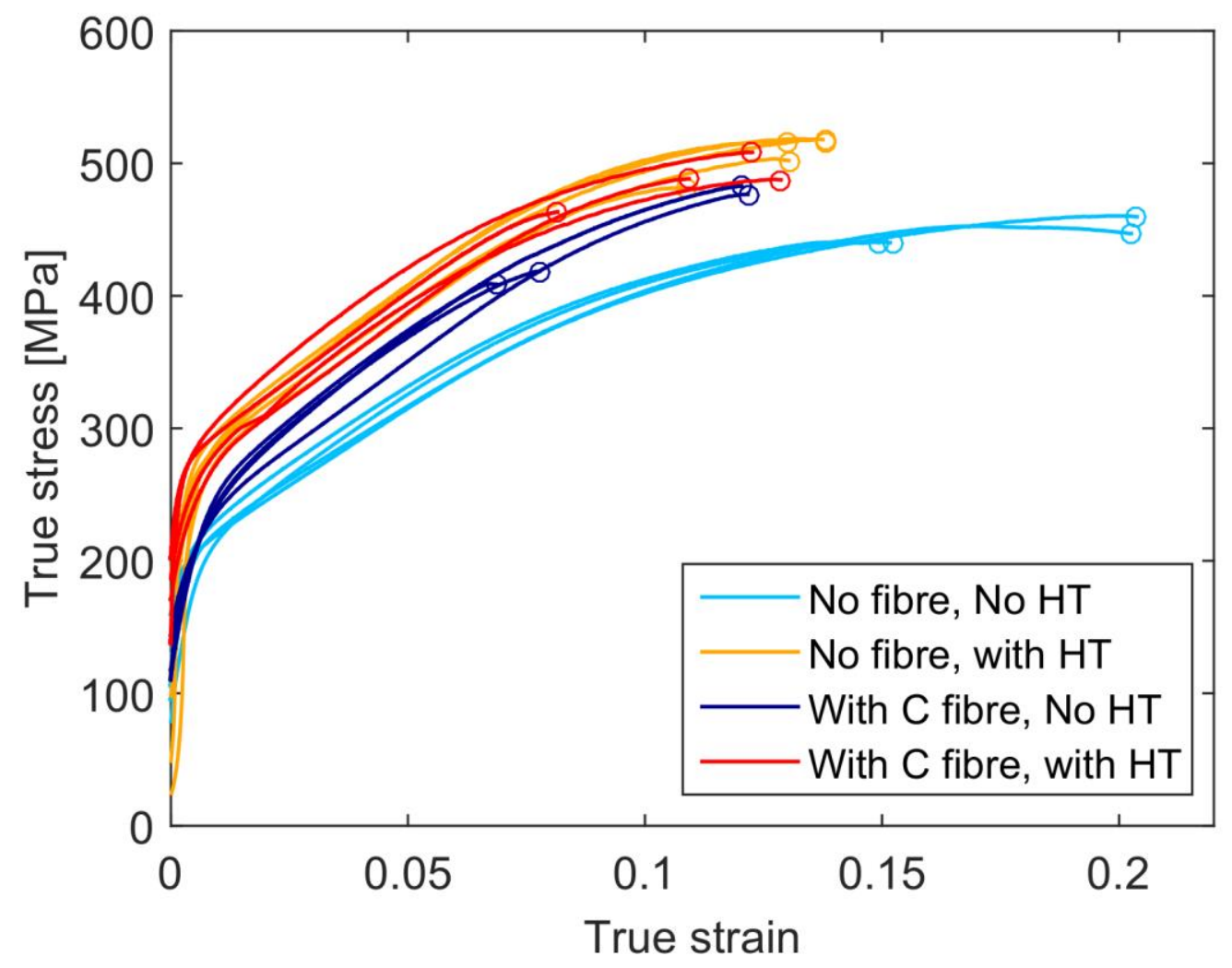

Figure 5. Experimental true stress-strain curves of the four FSP materials in compression: Reference (unreinforced) and composite samples with and without heat treatment. The curves are represented up to the final fracture and the fracture stress and strain are represented by a circle. 


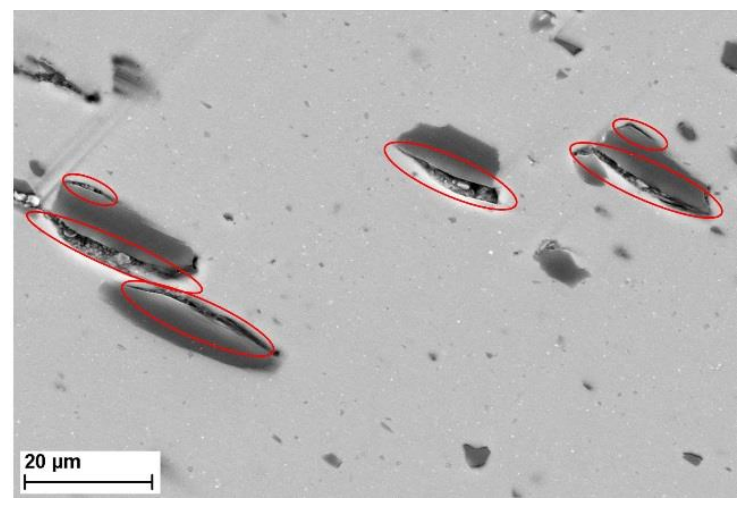

(a)

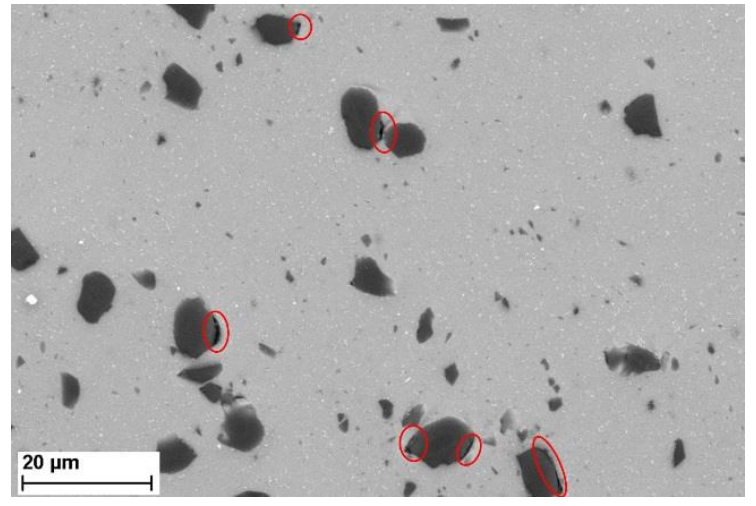

(b)

Figure 6. SEM micrographs of (a) the tensile and (b) the compression samples without heat treatment after failure observed a few millimetres below the fracture surface and at midthickness of the sample. The loading direction is vertical. Observed debonding between the matrix and the fibres is circled in red. 


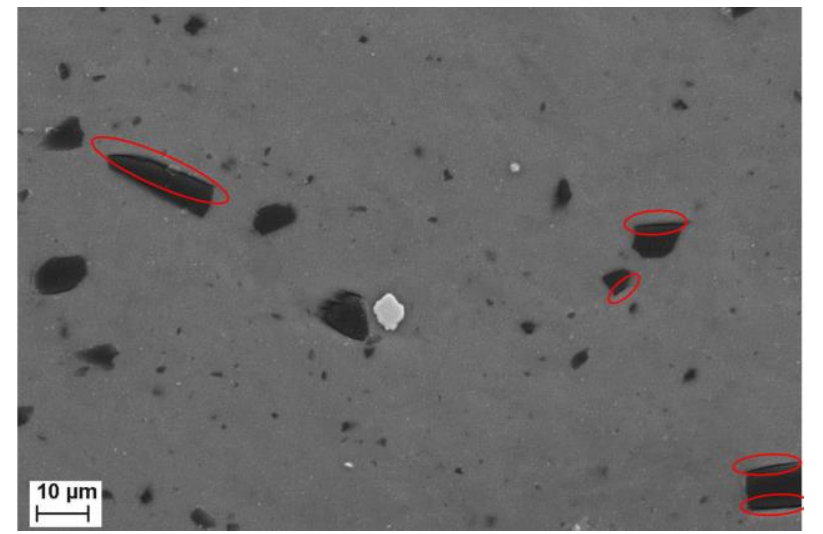

(a)

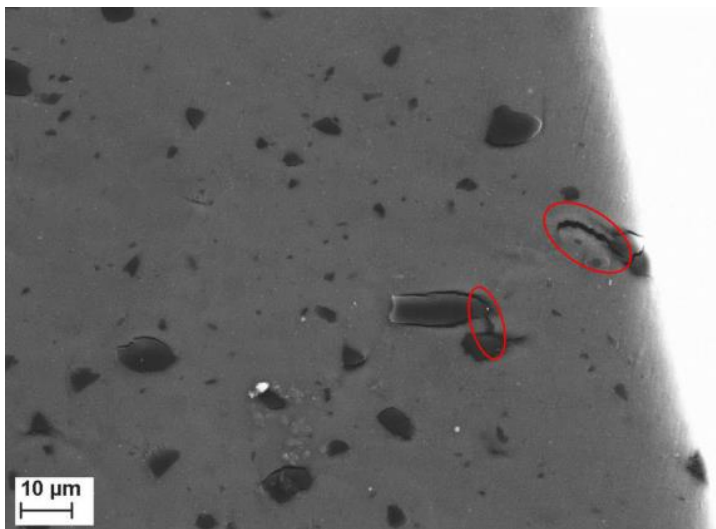

(b)

Figure 7. SEM micrographs from an in-situ tensile notched specimen showing (a) damage nucleation due to fibre-matrix debonding and (b) the onset of void coalescence. For better visibility the debonding and coalescence are highlighted by red contours in (a) and (b) respectively. 


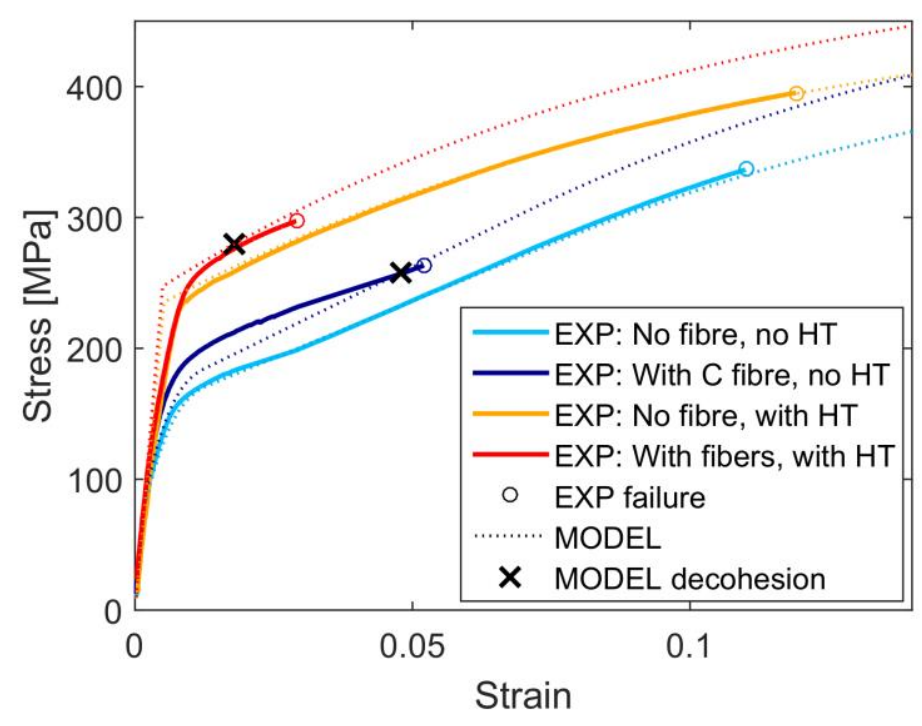

(a)

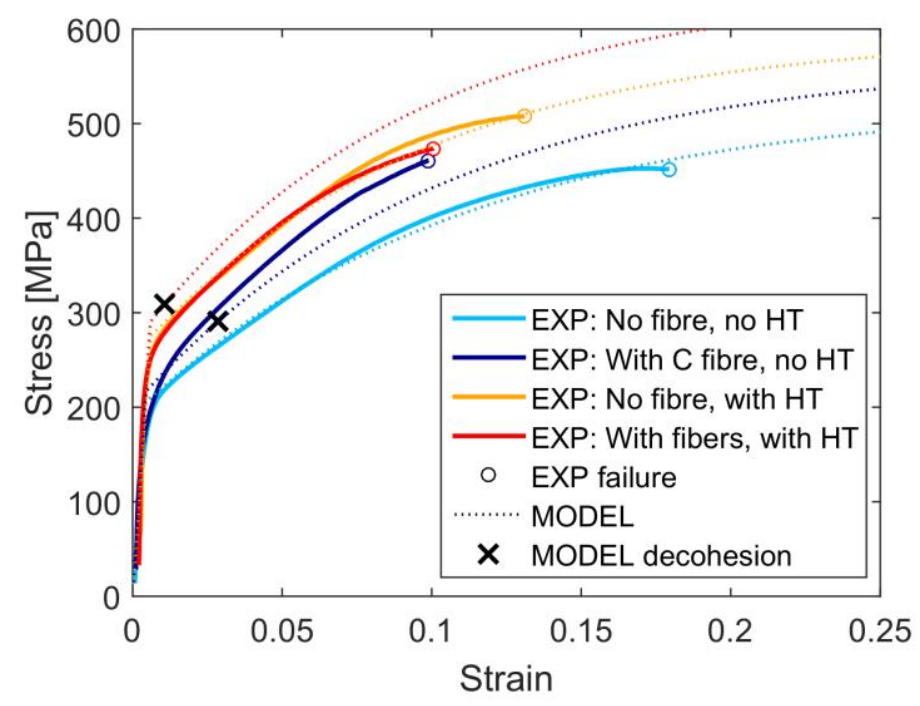

(b)

Figure 8. True stress-strain curves for the FSP materials with or without HT, and with or without fibres, in (a) tension and (b) compression. Model predictions (dashed lines) are compared to reference experimental curves (solid lines). The black crosses indicate the onset of interfacial debonding, according to the micromechanical model while coloured open circles are the experimental fracture. The experimental curves are representative curves having the same yield strength and deformation at failure as the mean value (see Table 1) of all tested samples. 


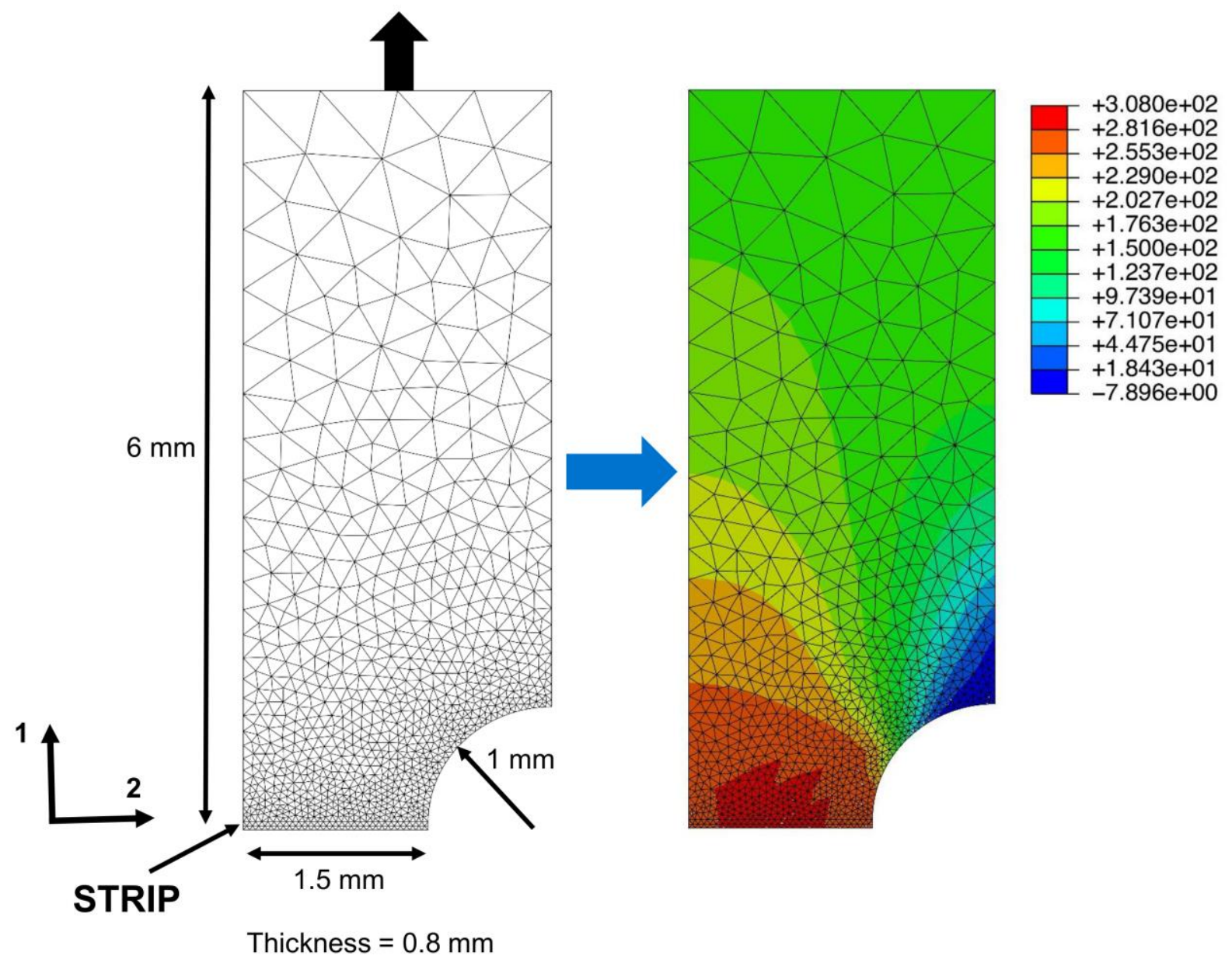

Figure 9. Mesh used for the simulation of the in-situ notched tensile test (left) and macroscopic stress in the loading direction $\bar{\sigma}_{11}$, predicted by the micromechanical model at the onset of interface debonding (based on SEM observations, Figure 7a). The stress scale is in MPa. 


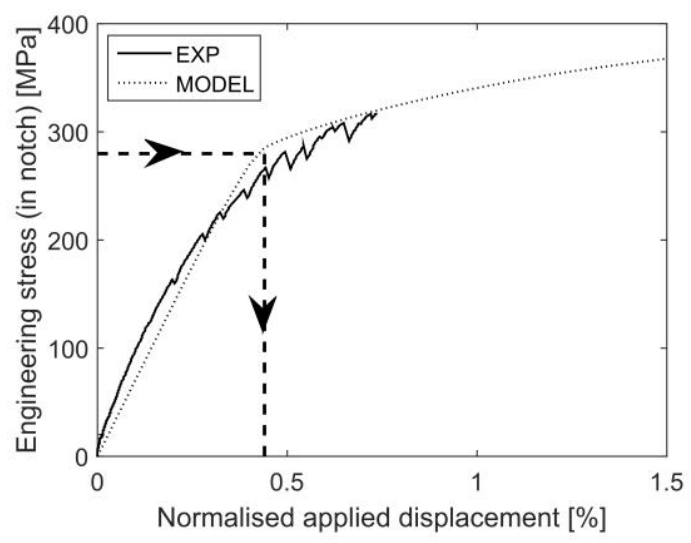

(a)

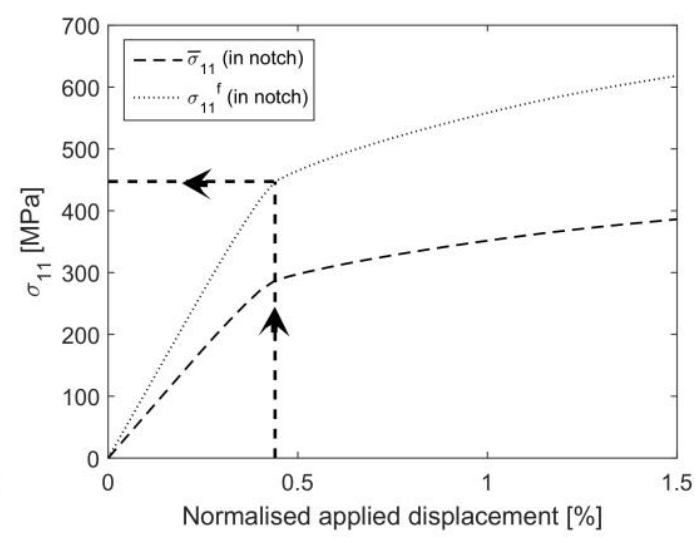

(b)

Figure 10. (a) Experimental and predicted engineering stress in the notch (applied force divided by the notch reduced section) as a function of the normalised applied displacement. The dashed arrowed line indicates the onset of interface debonding, based on SEM observations. (b) Average fibre stress $\sigma_{11}^{f}$ and macroscopic stress $\bar{\sigma}_{11}$ in the notch (volume average over the strip region shown in Figure 9), as predicted by the model, as a function of the normalised applied displacement. The fibre stress at the onset of debonding is found to be $435 \mathrm{MPa}$. 


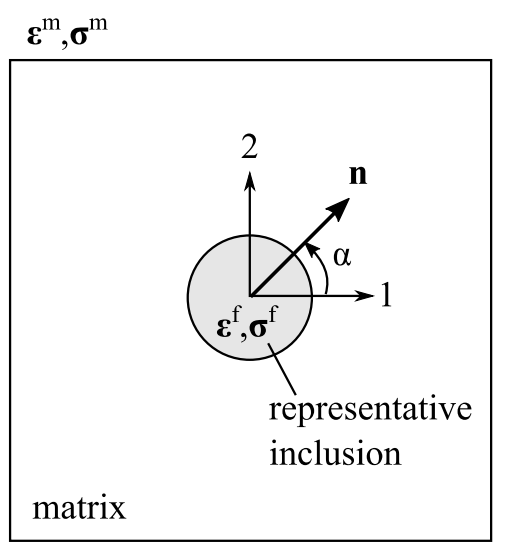

(a)

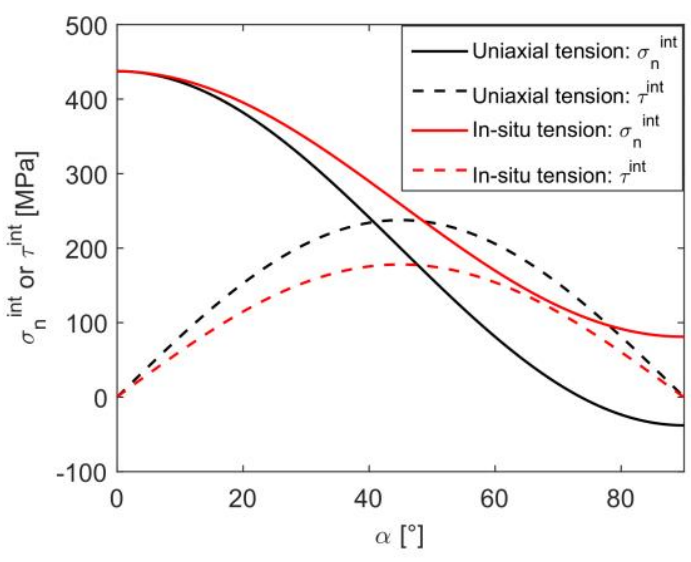

(b)

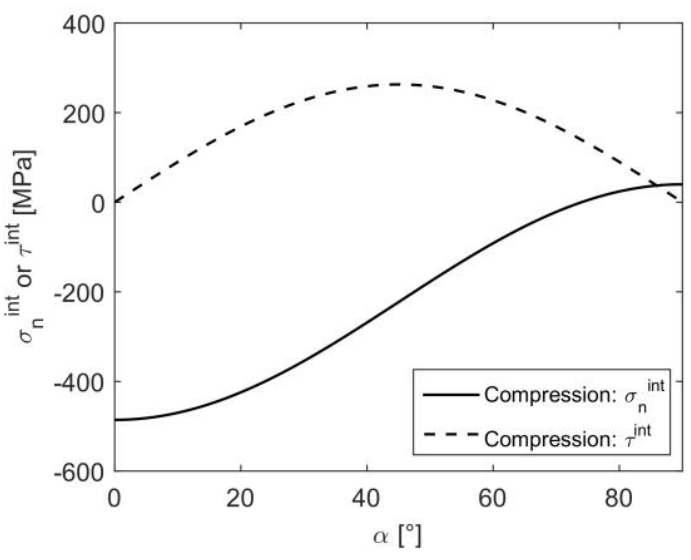

(c)

Figure 11. (a) According to the Mori-Tanaka scheme, the fibre phase is represented by a representative spherical inclusion embedded in an infinite matrix subjected to the matrix average strain as far-field strain. The orientation of a plane tangent to the matrix-fibre interface is described by its unit normal in the 1-2 plane making angle $\alpha$ with axis 1 (macroscopic loading direction). (b)-(c) Evolution of the interfacial normal stress and resultant shear stress as a function of the normal orientation in the 1-2 plane at the onset of debonding, under (a) tension and (b) compression, in the composites with HT. (a) In tension, debonding is predicted to occur when the normal stress reaches $\sigma_{c}=435 \mathrm{MPa}$, based on the in-situ tensile tests on a notched sample. (b) The profiles in compression correspond to the loading step where the maximum shear stress reaches the lower bound for the critical shear stress: $\tau_{c}=0.6 \sigma_{c}=250 \mathrm{MPa}$. 


\section{TABLES}

Table 1. Hardness extracted from the Berkovich indentation tests for samples with or without $\mathrm{C}$ fibers and with or without a post-FSP heat treatment at $200^{\circ} \mathrm{C}$ for $8 \mathrm{~h}(\mathrm{HT})$.

\begin{tabular}{|l|c|}
\hline & Hardness [GPa] \\
\hline No fibers, No HT & $1.27 \pm 0.05$ \\
\hline With C fibers, No HT & $1.35 \pm 0.05$ \\
\hline No Fibers, with HT & $1.40 \pm 0.05$ \\
\hline With C Fibers, with HT & $1.41 \pm 0.04$ \\
\hline
\end{tabular}


Table 2. Tensile and compression properties of the samples extracted from the FSP zone of samples with or without $\mathrm{C}$ fibers and with or without a post-FSP heat treatment (HT) at $200^{\circ} \mathrm{C}$ for $8 \mathrm{~h}$.

\begin{tabular}{|l|c|c|c|c|c|c|}
\hline & \multicolumn{3}{|c|}{ Tension } & \multicolumn{3}{c|}{ Compression } \\
\cline { 2 - 7 } & $\sigma_{y 0}$ & $\sigma_{\text {max }}$ & $\varepsilon_{\text {max }}$ & $\sigma_{y 0}$ & $\sigma_{\text {max }}$ & $\varepsilon_{\text {max }}$ \\
& {$[$ MPa] } & {$[$ MPa] } & & {$[$ MPa $]$} & {$[$ MPa $]$} & \\
\hline No fibers, No HT & 151 & 329 & 0.110 & 205 & 449 & 0.177 \\
& \pm 3 & \pm 68 & \pm 0.051 & \pm 5 & \pm 10 & \pm 0.031 \\
\hline With C fibers, No HT & 173 & 266 & 0.052 & 247 & 447 & 0.097 \\
& \pm 9 & \pm 41 & \pm 0.034 & \pm 30 & \pm 38 & \pm 0.028 \\
\hline No Fibers, with HT & 236 & 403 & 0.119 & 275 & 508 & 0.129 \\
& \pm 14 & \pm 16 & \pm 0.014 & \pm 6 & \pm 16 & \pm 0.012 \\
\hline With C Fibers, with & 242 & 291 & 0.029 & 273 & 469 & 0.098 \\
HT & \pm 6 & \pm 7 & \pm 0.008 & \pm 19 & \pm 37 & \pm 0.026 \\
\hline
\end{tabular}

Table 3. True strain at matrix-fiber debonding predicted by the mean field model.

\begin{tabular}{|l|c|c|}
\hline & Under tension & Under compression \\
\hline With C fibers, No HT & 0.048 & 0.029 \\
\hline With C Fibers, with HT & 0.018 & 0.011 \\
\hline
\end{tabular}

